\title{
Detectability Subspaces and Observer Synthesis for Two-Dimensional Systems *
}

\author{
Lorenzo Ntogramatzidis ${ }^{\star} \quad$ Michael Cantoni ${ }^{\dagger}$ \\ ${ }^{\star}$ Department of Mathematics and Statistics, \\ Curtin University of Technology, Perth, 6845 WA, Australia. \\ E-mail: L.Ntogramatzidis@curtin.edu.au \\ †Department of Electrical and Electronic Engineering, \\ The University of Melbourne, Parkville, 3010 VIC, Australia. \\ E-mail: cantoni@unimelb.edu.au
}

\begin{abstract}
The notions of input-containing and detectability subspaces are developed within the context of observer synthesis for two-dimensional (2-D) Fornasini-Marchesini models. Specifically, the paper considers observers which asymptotically estimate the local state, in the sense that the error tends to zero as the reconstructed local state evolves away from possibly mismatched boundary values, modulo a detectability subspace. Ultimately, the synthesis of such observers in the absence of explicit input information is addressed.
\end{abstract}

Keywords: Fornasini-Marchesini models; Detectability subspaces; Observers; Unknown-Input Observation.

\section{Introduction}

Controlled invariant subspaces were originally introduced by Basile and Marro in [1] to solve decoupling and tracking problems for one-dimensional (1-D) systems. These subspaces were subsequently studied by Wonham and Morse in [33]. Conditioned invariant subspaces for 1-D systems were also introduced by Basile and Marro in [1], as the duals of controlled invariant subspaces. The role of such subspaces in solving state estimation problems was first investigated in [2]. Later, conditioned invariance was studied by Willems in terms of the existence of a class of observers [32]. Specifically, for any conditioned invariant subspace $\mathcal{S}$ that can be externally stabilised by output-injection, there exists an observer that asymptotically recovers the state modulo $\mathcal{S}$; see also the textbooks [3, Chapter 4] and [30, Chapter 5]. Unlike the classic Luenberger observer, this class of observers does not directly

${ }^{*}$ This work was partially supported by the Australian Research Council (Discovery Grant DP0986577). 
exploit the input in reconstructing the state. The relevance of this for problems in fault-detection and isolation is well established, [25, 31].

Over the last twenty years, several extensions of important geometric concepts, such as controlled invariance, have been proposed for 2-D systems, including the so-called Fornasini-Marchesini and Roesser models, [9, 20, 21]. While definitions of controlled invariance are not difficult to establish for Fornasini-Marchesini models, definitions of conditioned invariance are less obvious because duality cannot be exploited as in the 1-D case. The definitions of conditioned invariance in $[20,21]$ have the disadvantage of being defined for models with very special structures; that is, with duality properties absent in the case of the standard Fornasini-Marchesini model. In [9], a definition of conditioned invariance is proposed for the standard (first order) Fornasini-Marchesini model [13], governed by

$$
\begin{aligned}
x_{i+1, j+1} & =A_{1} x_{i+1, j}+A_{2} x_{i, j+1}+B_{1} u_{i+1, j}+B_{2} u_{i, j+1}, \\
y_{i, j} & =C x_{i, j}+D u_{i, j} .
\end{aligned}
$$

The definition of conditioned invariance in [9] is related therein to the reconstruction of local state trajectories given a record of the output, in the case of exact knowledge of the boundary conditions for (1). The problem of local state estimation with decaying error in the case of unknown boundary conditions has not been considered. Motivated by this, we develop a systematic procedure for the external stabilisation of conditioned invariant subspaces via output injections. This will lead to a notion of 2-D detectability subspaces, i.e. conditioned invariant subspace that can be externally stabilised by output injection. An algorithm for computing a stabilising output injection matrix is also provided in terms of matrix inequalities. The approach is similar to [27], where internal stabilisability of output-nulling controlled invariant subspaces is studied. Ultimately, the notion of a detectabilty subspace is linked to the existence of a local state observer

$$
\omega_{i+1, j+1}=K_{1} \omega_{i+1, j}+K_{2} \omega_{i, j+1}+L_{1} y_{i+1, j}+L_{2} y_{i, j+1}
$$

It is required that the size of the estimation error $e_{i, j} \stackrel{\text { def }}{=} Q x_{i, j}-\omega_{i, j}$ asymptotically approaches zero as $i+j \rightarrow \infty$, for some full row-rank matrix $Q$ and arbitrary boundary conditions for $x_{i, j}$ and $\omega_{i, j}$. The local state observer (2), like its 1-D counterparts defined in [32], does not exploit knowledge of the input. As such, it is structurally different from the Luenberger-type 2-D observers discussed in [4], since its local state does not explicitly depend on the input $u_{i, j}$. For more details on the synthesis of 2-D observers, see also $[17,18]$ and the references therein. As an important application of the concepts described above, we present a solution to the so-called unknown-input observation problem for the standard 2-D system model (2), based on a sufficient constructive condition given in terms of a standard subspace inclusion. The relevance in fault detection and non-interaction are well known, $[6,7]$. In [5], a polynomial approach is employed to develop necessary and sufficient conditions for the solution of this problem under the requirement that the observer error exhibit dead-beat dynamics. The conditions involve Bézout equations, which can be difficult to solve. In this paper, the unknowninput observation problem is considered under the weaker requirement that the estimation error only asymptotically converge to zero away from the location of the unknown boundary conditions. 
Notation. Throughout this paper, we denote by $\mathbb{N}$ the set of positive integers including zero. The image and the kernel of matrix $M \in \mathbb{R}^{n \times m}$ are denoted by im $M$ and ker $M$, respectively. The $n \times m$ zero matrix is denoted by $0_{n \times m}$. We also denote by $M^{\top}$ and by $M^{\dagger}$ the transpose and the MoorePenrose pseudoinverse of $M$, respectively. Given a subspace $\mathcal{Y}$ of $\mathbb{R}^{m}$, the symbol $M^{-1} \mathcal{Y}$ stands for the inverse image of $\mathcal{Y}$ with respect to the linear transformation $M$. For the sake of brevity, we define $M_{D}:=\operatorname{diag}(M, M)$, and, accordingly, given a subspace $\mathcal{J}$ of $\mathbb{R}^{n}$, the symbol $\mathcal{J}_{D}$ will identify the subspace $\mathcal{J} \oplus \mathcal{J}$ of $\mathbb{R}^{2 n}$. Finally, given the vector $\xi \in \mathbb{R}^{n}$, the symbol $\xi / \mathcal{J}$ denotes the canonical projection of $\xi$ on the quotient space $\mathbb{R}^{n} / \mathcal{J}$.

\section{Invariant Subspaces for Fornasini-Marchesini Models}

We begin by recalling some preliminary geometric concepts for the autonomous Fornasini-Marchesini (FM) model

$$
x_{i+1, j+1}=A_{1} x_{i+1, j}+A_{2} x_{i, j+1},
$$

where, for all integers $i, j$, the vector $x_{i, j} \in \mathbb{R}^{n}$ is the local state of the system. Here, $A_{1}, A_{2} \in \mathbb{R}^{n \times n}$. Defining for each $k \in \mathbb{Z}$ the separation set

$$
\mathbb{S}_{k} \stackrel{\text { def }}{=}\{(i, j) \in \mathbb{Z} \times \mathbb{Z} \mid i+j=k\},
$$

and the corresponding instance of the global state

$$
\mathcal{X}_{k} \stackrel{\text { def }}{=}\left\{x_{i, j} \in \mathbb{R}^{n} \mid(i, j) \in \mathbb{S}_{k}\right\},
$$

it is easily seen from (3) that $\mathcal{X}_{k}$ can be uniquely expressed in terms of $\mathcal{X}_{k-1}$, [13]. In particular, if we fix the values of $x_{i, j}$ on $\mathbb{S}_{0}$, i.e. fix $\mathcal{X}_{0}$ as a boundary condition, (3) uniquely determines $\mathcal{X}_{k}$ for $k>0$ (i.e., $x_{i, j}$ for $\left.i+j>0\right) .{ }^{1}$ Indeed, these are the boundary conditions usually associated with the FM model (3). In the sequel, given a subspace $\mathcal{W} \subseteq \mathbb{R}^{n}$, by a $\mathcal{W}$-valued boundary condition we intend the set $\left\{x_{i, j} \in \mathcal{W} \mid(i, j) \in \mathbb{S}_{0}\right\}$. Similarly, for each $k>0$, the global state $\mathcal{X}_{k}$ is said to be $\mathcal{W}$-valued when $x_{i, j} \in \mathcal{W}$ for all $(i, j) \in \mathbb{S}_{k}$. We also define the set of indexes for which the local state $x_{i, j}$ of (3) is uniquely determined by fixing $\mathcal{X}_{0}$ as boundary condition:

$$
\mathbb{S}_{+} \stackrel{\text { def }}{=} \bigcup_{k \in \mathbb{N}} \mathbb{S}_{k}=\{(i, j) \in \mathbb{Z} \times \mathbb{Z} \mid i+j \geq 0\}
$$

A subspace $\mathcal{J}$ of $\mathbb{R}^{n}$ is said to be $\left(A_{1}, A_{2}\right)$-invariant if $\mathcal{J}$ is $A_{i}$-invariant in the usual 1-D sense for $i \in\{1,2\}$; i.e., $A_{i} x \in \mathcal{J}$ for all $x \in \mathcal{J}$ and $i \in\{1,2\}$. The notation $A_{i} \mathcal{J} \subseteq \mathcal{J}$ is also commonly used

\footnotetext{
${ }^{1}$ As shown in [14], other separation sets can be defined so that boundary conditions specified over them uniquely determine a local-state trajectory solution of (3) over a region of $\mathbb{Z} \times \mathbb{Z}$. An interesting and useful example is the separation set $\mathbb{S}_{k} \stackrel{\text { def }}{=}\{(i, j) \in\{0\} \times[1, \infty) \cup[1, \infty) \times\{0\}\}$, which with corresponding boundary conditions uniquely determines $x_{i, j}$ for $(i, j) \in \mathbb{S}_{+} \stackrel{\text { def }}{=} \mathbb{N} \times \mathbb{N} \backslash\{(0,0)\}$. Most of the considerations in this paper can be adapted to such separations sets.
} 
to denote this property. For example, the subspaces $\{0\}$ and $\mathbb{R}^{n}$ are $\left(A_{1}, A_{2}\right)$-invariant for every $n \times n$ matrices $A_{1}$ and $A_{2}$. The following lemma provides geometric and matrix conditions for invariance.

Lemma 2.1 The following are equivalent:

1) $\mathcal{J}$ is $\left(A_{1}, A_{2}\right)$-invariant;

2) $\left[\begin{array}{ll}A_{1} & A_{2}\end{array}\right](\mathcal{J} \oplus \mathcal{J}) \subseteq \mathcal{J}$, i.e., $A_{1} x+A_{2} y \in \mathcal{J}$ for all $x, y \in \mathcal{J}$;

3) There exist $L_{1}, L_{2} \in \mathbb{R}^{(n-r) \times(n-r)}$ such that $Q A_{i}=L_{i} Q$ for $i \in\{1,2\}$, i.e., $Q\left[A_{1} \quad A_{2}\right]=$ $\left[\begin{array}{ll}L_{1} & L_{2}\end{array}\right] Q_{D}$, where $Q \in \mathbb{R}^{(n-r) \times n}$ is a full row-rank matrix such that $\operatorname{ker} Q=\mathcal{J}$.

Proof: 1) $\Leftrightarrow$ 2) Follows straightforwardly from the 1-D counterpart, [3].

2) $\Leftrightarrow 3)$ Note that 2) is equivalent to $\operatorname{ker} Q_{D} \subseteq \operatorname{ker}\left(Q\left[\begin{array}{ll}A_{1} & A_{2}\end{array}\right]\right)$, by which the result holds, since for any matrices $M \in \mathbb{R}^{p \times m}$ and $N \in \mathbb{R}^{q \times p}$, ker $M \subseteq$ ker $N$ if and only if an $L \in \mathbb{R}^{q \times m}$ exists such that $N=L M$.

The following theorem is the 2-D counterpart of a fundamental result on $A$-invariance (see [1]), concerning the decomposition of a 1-D system matrix with respect to an invariant subspace.

Theorem 2.1 Let $\mathcal{J}$ be an $r$-dimensional subspace of $\mathbb{R}^{n}$. The following statements are equivalent:

1) The subspace $\mathcal{J}$ is $\left(A_{1}, A_{2}\right)$-invariant;

2) With respect to any basis in $\mathbb{R}^{n}$ whose first $r$ vectors span $\mathcal{J}$, the linear transformations $A_{1}$ and $A_{2}$ are given respectively by the block-triangular matrices

$$
\left[\begin{array}{cc}
A_{1}^{11} & A_{1}^{12} \\
0_{(n-r) \times r} & A_{1}^{22}
\end{array}\right] \quad \text { and } \quad\left[\begin{array}{cc}
A_{2}^{11} & A_{2}^{12} \\
0_{(n-r) \times r} & A_{2}^{22}
\end{array}\right] \text {. }
$$

The proof is a straightforward consequence of Lemma 2.1, see also the proof of Theorem 2.1 in [27].

\subsection{Invariant Subspaces and Local-State Trajectories}

In this section the concept of $\left(A_{1}, A_{2}\right)$-invariance is used to analyse properties of the local state trajectories generated by (3). Consider an $\left(A_{1}, A_{2}\right)$-invariant subspace $\mathcal{J}$. A boundary condition $\left\{x_{i, j}=b_{i, j} \in \mathcal{J} \mid(i, j) \in \mathbb{S}_{0}\right\}$ gives rise to $x_{i, j} \in \mathcal{J}$ for all $(i, j) \in \mathbb{S}_{+}$. In fact, in view of Theorem 2.1, a similarity transformation $S \in \mathbb{R}^{n \times n}$ exists such that for each $i \in\{1,2\}$ there holds

$$
\hat{A}_{i} \stackrel{\text { def }}{=} S \hat{A}_{i} S^{-1}=\left[\begin{array}{cc}
\hat{A}_{i}^{11} & \hat{A}_{i}^{12} \\
0_{(n-r) \times r} & \hat{A}_{i}^{22}
\end{array}\right] .
$$

Matrix $S$ is any basis matrix of $\mathbb{R}^{n}$ adapted to $\mathcal{J}$, i.e., such that its first columns span $\mathcal{J}$. Equivalently, $S$ can be constructed as the square non-singular matrix $S=\left[\begin{array}{c}S_{c} \\ Q\end{array}\right]$, where $\operatorname{ker} Q=\mathcal{J}$ and the rows of 
$S_{c}$ are linearly independent from those of $Q$. With respect to this new set of coordinates, model (3) can be written as

$$
\left[\begin{array}{l}
x_{i+1, j+1}^{\prime} \\
x_{i+1, j+1}^{\prime \prime}
\end{array}\right]=\left[\begin{array}{cc}
\hat{A}_{1}^{11} & \hat{A}_{1}^{12} \\
0 & \hat{A}_{1}^{22}
\end{array}\right]\left[\begin{array}{c}
x_{i+1, j}^{\prime} \\
x_{i+1, j}^{\prime \prime}
\end{array}\right]+\left[\begin{array}{cc}
\hat{A}_{2}^{11} & \hat{A}_{2}^{12} \\
0 & \hat{A}_{2}^{22}
\end{array}\right]\left[\begin{array}{c}
x_{i, j+1}^{\prime} \\
x_{i, j+1}^{\prime \prime}
\end{array}\right]
$$

Any boundary condition $\left\{x_{i, j}=b_{i, j} \in \mathcal{J} \mid(i, j) \in \mathbb{S}_{0}\right\}$ is such that $x_{i, j}^{\prime \prime}=0$ for $(i, j) \in \mathbb{S}_{0}$. Therefore, by (5), $x_{i, j}^{\prime \prime}=0$ for all $(i, j) \in \mathbb{S}_{+}$, which implies that $x_{i, j} \in \mathcal{J}$ for all $i, j \in \mathbb{S}_{+}$. In the basis corresponding to $S$, whereby $\left[\begin{array}{l}x_{i, j}^{\prime} \\ x_{i, j}^{\prime \prime}\end{array}\right]=S x_{i, j}$, the component $x_{i, j}^{\prime}$ is the projection of the local state $x_{i, j}$ onto the invariant subspace $\mathcal{J}$, while $x_{i, j}^{\prime \prime}$ is the canonical projection on to the quotient space $\mathbb{R}^{n} / \mathcal{J}$. Thus, we refer to $x_{i, j}^{\prime}$ of $x_{i, j}$ as the internal (or inner) component of the local state (with respect to $\mathcal{J}$ ), and to $x_{i, j}^{\prime \prime}$ of $x_{i, j}$ as the external (or outer) component of the local state (with respect to $\mathcal{J})$.

\subsection{Internal and External Stability of Invariant Subspaces}

With $\left\|\mathcal{X}_{k}\right\| \stackrel{\text { def }}{=} \sup _{n \in \mathbb{Z}}\left\|x_{k-n, n}\right\|$, the system model (3) is said to be asymptotically stable if for any boundary condition satisfying $\left\|\mathcal{X}_{0}\right\|<\infty$, the corresponding sequence $\left\{\left\|\mathcal{X}_{i}\right\|\right\}_{i=0}^{\infty}$ converges to zero [13]. This property is clearly invariant under coordinate transformation and with a slight abuse of nomenclature, the system matrix pair $\left(A_{1}, A_{2}\right)$ is called asymptotically stable in this case. It is well-known that the pair $\left(A_{1}, A_{2}\right)$ is asymptotically stable if, and only if,

$$
\operatorname{det}\left(I_{n}-A_{1} z_{2}-A_{2} z_{1}\right) \neq 0 \quad \forall\left(z_{1}, z_{2}\right) \in \mathfrak{P}
$$

where $\mathfrak{P}=\left\{\left(\zeta_{1}, \zeta_{2}\right) \in \mathbb{C} \times \mathbb{C}|| \zeta_{1} \mid \leq 1\right.$ and $\left.\left|\zeta_{2}\right| \leq 1\right\}$ is the unit bidisc [13, Proposition 3]. Various, more computationally tractable, sufficient stability conditions have been proposed over the last two decades, in terms of Lyapunov equations and/or spectral radius conditions of certain matrices, see e.g. $[19,8]$. In the very recent literature, new necessary and sufficient criteria have appeared for asymptotic stability in terms of conditions that have a more complex structure, but that can be checked in finite time, see [34, 12]. For the sake of argument and clarity, however, we limit ourselves to recalling and using the following simple sufficient condition for asymptotic stability, expressed in terms of a linear matrix inequality (LMI):

Lemma 2.2 ([19]) The pair $\left(A_{1}, A_{2}\right)$ is asymptotically stable if two symmetric positive definite matrices $P_{1}$ and $P_{2}$ exist such that:

$$
\operatorname{diag}\left(P_{1}, P_{2}\right)-\left[\begin{array}{c}
A_{1}^{\top} \\
A_{2}^{\top}
\end{array}\right]\left(P_{1}+P_{2}\right)\left[\begin{array}{ll}
A_{1} & A_{2}
\end{array}\right]>0 .
$$

The LMI condition in Lemma 2.2 is one of the most utilised for analysis and synthesis problems involving FM models. Here it is used to develop a procedure for the computation of output injection matrices that stabilise the external dynamics of conditioned invariant and input-containing subspaces, which are defined shortly. 
As shown in [27], the stability of (3) can be studied in terms of two parts, with respect to a given $\left(A_{1}, A_{2}\right)$-invariant subspace $\mathcal{J}$. In particular, in the set of coordinates of $(5)$, the fact that

$$
\operatorname{det}\left(I_{n}-\hat{A}_{1} z_{2}-\hat{A}_{2} z_{1}\right)=\operatorname{det}\left(I-\hat{A}_{1}^{11} z_{2}-\hat{A}_{2}^{11} z_{1}\right) \cdot \operatorname{det}\left(I-\hat{A}_{1}^{22} z_{2}-\hat{A}_{2}^{22} z_{1}\right)
$$

implies that (3) is asymptotically stable if and only if the two matrix pairs $\left(\hat{A}_{1}^{11}, \hat{A}_{2}^{11}\right)$ and $\left(\hat{A}_{1}^{22}, \hat{A}_{2}^{22}\right)$ are each asymptotically stable. Moreover, when a $\mathcal{J}$-valued boundary condition is imposed, for all $k \geq 0$ the global state $\mathcal{X}_{k}^{\prime \prime}$ associated with the external dynamics (5) satisfies $\left\|\mathcal{X}_{k}^{\prime \prime}\right\|=0$. Hence, the internal dynamics on $\mathcal{J}$ satisfy

$$
x_{i+1, j+1}^{\prime}=\hat{A}_{1}^{11} x_{i+1, j}^{\prime}+\hat{A}_{2}^{11} x_{i, j+1}^{\prime} .
$$

If $\left(\hat{A}_{1}^{11}, \hat{A}_{2}^{11}\right)$ alone is also asymptotically stable, then the global state $\mathcal{X}_{k}^{\prime}$ associated with (9) satisfies $\left\|\mathcal{X}_{k}^{\prime}\right\| \rightarrow 0$, and therefore also $\left\|\mathcal{X}_{k}\right\| \rightarrow 0$.

Definition 2.1 The r-dimensional $\left(A_{1}, A_{2}\right)$-invariant subspace $\mathcal{J}$ is said to be

internally stable if the internal dynamics governed by (9) are asymptotically stable; i.e., the corresponding pair $\left(\hat{A}_{1}^{11}, \hat{A}_{2}^{11}\right)$ is asymptotically stable;

externally stable if the external dynamics governed by (5) are asymptotically stable; i.e., the corresponding pair $\left(\hat{A}_{1}^{22}, \hat{A}_{2}^{22}\right)$ is asymptotically stable.

Consider now a boundary condition that is not $\mathcal{J}$-valued, so that $\left\|\mathcal{X}_{0}^{\prime \prime}\right\| \neq 0$. It follows from (5) that $\left\|\mathcal{X}_{k}^{\prime \prime}\right\| \rightarrow 0$ if, and only if, the pair $\left(\hat{A}_{1}^{22}, \hat{A}_{2}^{22}\right)$ is asymptotically stable, and in this case, the elements of the global state $\mathcal{X}_{k}$ associated with (3) approach the invariant subspace $\mathcal{J}$, as $k \rightarrow \infty$. Finally, in view of the discussion above, note that the model (3) is asymptotically stable if, and only if, any $\left(A_{1}, A_{2}\right)$-invariant subspace is both internally and externally stable. The following corollary will be important in the sequel.

Corollary 2.1 Given an $r$-dimensional subspace $\mathcal{J}$ of $\mathbb{R}^{n}$, let $Q \in \mathbb{R}^{(n-r) \times n}$ be a full row-rank matrix such that $\operatorname{ker} Q=\mathcal{J}$. Then $\mathcal{J}$ is an externally stable $\left(A_{1}, A_{2}\right)$-invariant subspace if, and only if, an asymptotically stable pair $\left(L_{1}, L_{2}\right)$ exists such that $Q A_{i}=L_{i} Q$ for $i \in\{1,2\}$.

Proof: With respect to a basis of $\mathbb{R}^{n}$ adapted to $\mathcal{J}$, a matrix whose columns span $\mathcal{J}$ is $\left[\begin{array}{c}I_{r \times r} \\ 0_{(n-r) \times r}\end{array}\right]$, and therefore $Q=\left[\begin{array}{ll}0_{(n-r) \times r} & I_{(n-r)}\end{array}\right]$ is a full row-rank matrix such that $\operatorname{ker} Q=\mathcal{J}$. Writing the identities $Q A_{i}=L_{i} Q$ for $i \in\{1,2\}$ with respect to this basis yields

$$
\left[\begin{array}{ll}
0_{(n-r) \times r} & I_{n-r}
\end{array}\right]\left[\begin{array}{cc}
A_{i}^{11} & A_{i}^{12} \\
0_{(n-r) \times r} & A_{i}^{22}
\end{array}\right]=L_{i}\left[\begin{array}{ll}
0_{(n-r) \times r} & I_{n-r}
\end{array}\right],
$$

leading to $L_{i}=A_{i}^{22}$ for all $i \in\{1,2\}$. 


\section{Conditioned Invariant Subspaces}

Consider the Fornasini-Marchesini model (1) where, for all integers $i, j$, vector $x_{i, j} \in \mathbb{R}^{n}$ is the local state, $u_{i, j} \in \mathbb{R}^{m}$ is the control input, $y_{i, j} \in \mathbb{R}^{p}$ is the output, $A_{k} \in \mathbb{R}^{n \times n}$ and $B_{k} \in \mathbb{R}^{n \times m}$ for $k \in\{1,2\}$, $C \in \mathbb{R}^{p \times n}$ and $D \in \mathbb{R}^{p \times m}$.

Definition 3.1 ([9]) The subspace $\mathcal{S} \subseteq \mathbb{R}^{n}$ is conditioned invariant for (1) if

$$
A_{H}\left(\mathcal{S}_{D} \cap \operatorname{ker} C_{D}\right) \subseteq \mathcal{S},
$$

where $A_{H} \stackrel{\text { def }}{=}\left[\begin{array}{ll}A_{1} & A_{2}\end{array}\right], C_{D} \stackrel{\text { def }}{=} \operatorname{diag}(C, C)$ and $S_{D}=S \oplus S$.

As for the 1-D case, it is easily seen that the set of conditioned invariant subspaces is closed under subspace intersection but not under subspace addition. Its smallest element (with respect to the partial relation of subspace inclusion $\subseteq$ ) is $\{0\}$, its largest element is $\mathbb{R}^{n}$. In the following lemma, the most important properties of 2-D conditioned invariance are given.

Lemma 3.1 Let $\mathcal{S}$ be an $s$-dimensional subspace of $\mathbb{R}^{n}$, and let $Q \in \mathbb{R}^{(n-s) \times n}$ be a full row-rank matrix such that $\operatorname{ker} Q=\mathcal{S}$. The following statements are equivalent:

1) the subspace $\mathcal{S}$ is conditioned invariant for (1);

2) there exist matrices $\Gamma=\left[\begin{array}{ll}\Gamma_{1} & \Gamma_{2}\end{array}\right]$ and $\Lambda=\left[\begin{array}{ll}\Lambda_{1} & \Lambda_{2}\end{array}\right]$ - with $\Gamma_{i} \in \mathbb{R}^{(n-s) \times(n-s)}$ and $\Lambda_{i} \in \mathbb{R}^{(n-s) \times p}$ for $i \in\{1,2\}-$ such that

$$
Q A_{H}=\Gamma Q_{D}+\Lambda C_{D}
$$

3) there exist a matrix $G=\left[\begin{array}{ll}G_{1} & G_{2}\end{array}\right]$ - with $G_{i} \in \mathbb{R}^{n \times p}$ - such that

$$
\left(A_{H}+G C_{D}\right) \mathcal{S}_{D} \subseteq \mathcal{S},
$$

Proof: 1) $\Longrightarrow$ 2). Inclusion (10) can be written in matrix notation as ker $\left[\begin{array}{l}Q_{D} \\ C_{D}\end{array}\right] \subseteq \operatorname{ker} Q\left[\begin{array}{ll}A_{1} & A_{2}\end{array}\right]$. Hence, matrices $\Gamma \in \mathbb{R}^{(n-s) \times 2(n-s)}$ and $\Lambda \in \mathbb{R}^{(n-s) \times 2 p}$ exist such that $Q\left[\begin{array}{ll}A_{1} & A_{2}\end{array}\right]=\Gamma Q_{D}+\Lambda C_{D}$.

2) $\Longrightarrow 3$ ). Equation (12) follows from (11) with any $G$ such that $\Lambda=-Q G$. Such a matrix $G$ always exists as $Q$ is of full row-rank.

3) $\Longrightarrow 1$ ). This follows from the definition.

Remark 3.1 Notice that property 2) in Lemma 3.1 can be written equivalently as

$$
Q A_{i}=\Gamma_{i} Q+\Lambda_{i} C \text { for } i \in\{1,2\} .
$$

As a consequence, inclusion (10) in Definition 3.1 can also be written as $A_{i}(\mathcal{S} \cap \operatorname{ker} C) \subseteq \mathcal{S}$ for $i \in\{1,2\}$, which coincides with the definition of 2-D conditioned invariance given in [9]. Moreover, 3) in Lemma 3.1 is equivalent to

$$
\left(A_{i}+G_{i} C\right) \mathcal{S} \subseteq \mathcal{S} \text { for } i \in\{1,2\} .
$$

This means that $\mathcal{S}$ is conditioned invariant for (1) if and only if there exists an output-injection matrix $G=\left[\begin{array}{ll}G_{1} & G_{2}\end{array}\right] \in \mathbb{R}^{n \times 2 p}$ such that $\mathcal{S}$ is an $\left(A_{1}+G_{1} C, A_{2}+G_{2} C\right)$-invariant subspace. 


\subsection{Construction of Stabilising Output-Injection Matrices}

Our aim now is to establish a procedure that enables an output-injection matrix $G$ to be determined such that $\mathcal{S}$ is an externally stable $\left(A_{1}+G_{1} C, A_{2}+G_{2} C\right)$-invariant subspace. As for the 1-D case, we say that $\mathcal{S}$ is externally stabilisable if we can find an output-injection matrix $G$ such that $\mathcal{S}$ is an externally stable $\left(A_{1}+G_{1} C, A_{2}+G_{2} C\right)$-invariant subspace.

To find all the output-injection matrices associated with the conditioned invariant subspace $\mathcal{S}$, let $\Gamma$ and $\Lambda$ be such that (11) holds, which can be written as the linear equation

$$
Q A_{H}=\left[\begin{array}{ll}
\Gamma & \Lambda
\end{array}\right]\left[\begin{array}{l}
Q_{D} \\
C_{D}
\end{array}\right] .
$$

This equation can be solved for $\Gamma$ and $\Lambda$. The solutions of (13) are given by

$$
\left[\begin{array}{ll}
\Gamma & \Lambda
\end{array}\right]=Q A_{H}\left[\begin{array}{l}
Q_{D} \\
C_{D}
\end{array}\right]^{\dagger}+K H
$$

where $H$ has linearly independent rows and $\operatorname{ker} H=\operatorname{im}\left[\begin{array}{l}Q_{D} \\ C_{D}\end{array}\right]$, while $K$ is an arbitrary matrix of suitable size. As it will become clear in the sequel, $K$ represents a first degree of freedom in the construction of the output-injection matrix, that can be exploited to externally stabilise the 2-D conditioned invariant subspace $\mathcal{S}$. Notice that in the case when $\left[\begin{array}{l}Q_{D} \\ C_{D}\end{array}\right]$ is full-rank, the only solution of (13) is $\left[\begin{array}{ll}\Gamma & \Lambda\end{array}\right]=Q A_{H}\left[\begin{array}{l}Q_{D} \\ C_{D}\end{array}\right]^{\dagger}$, and this degree of freedom disappears.

By (12), $\tilde{\Gamma}=\left[\begin{array}{ll}\tilde{\Gamma}_{1} & \tilde{\Gamma}_{2}\end{array}\right]$ exists such that

$$
Q\left(A_{H}+G C_{D}\right)=\tilde{\Gamma} Q_{D}
$$

or, equivalently, such that $Q\left(A_{i}+G_{i} C\right)=\tilde{\Gamma}_{i} Q$, for $i \in\{1,2\}$. We now investigate the relation between the pairs $(\Gamma, \Lambda)$ and $(G, \tilde{\Gamma})$ satisfying $(13)$ and $(15)$, respectively. Given a pair $(G, \tilde{\Gamma})$ such that (15) holds, then (13) is satisfied with $\Gamma=\tilde{\Gamma}$ and $\Lambda=-Q G$. Conversely, given a pair of matrices $(\Gamma, \Lambda)$ such that (13) holds, then (15) is satisfied with $\tilde{\Gamma}=\Gamma$ and with any $G$ such that $\Lambda=-Q G$. As such, no generality is lost by assuming $\tilde{\Gamma}=\Gamma$, and by representing the set of all output-injection matrices associated with the conditioned invariant subspace $\mathcal{S}$ as the set of matrices $G \in \mathbb{R}^{n \times 2 p}$ satisfying $\Lambda=-Q G$, where $\Lambda \in \mathbb{R}^{(n-s) \times 2 p}$ is any matrix for which $\Gamma \in \mathbb{R}^{(n-s) \times 2(n-s)}$ exists so that (13) holds. For any pair $(\Gamma, \Lambda)$ such that (13) holds, the solutions of the linear equation $\Lambda=-Q G$ are parameterised as

$$
G=G_{\Lambda}+\Omega U
$$

where $G_{\Lambda} \stackrel{\text { def }}{=}-Q^{\top}\left(Q Q^{\top}\right)^{-1} \Lambda$, matrix $\Omega$ is a basis of ker $Q$ and $U$ is an arbitrary matrix of suitable size. Hence, $U$ represents a second degree of freedom in the construction of the output-injection matrix associated with $\mathcal{S}$, that can be exploited to stabilise $\mathcal{S}$ internally. This second degree of 
freedom only disappears for $\mathcal{S}=\{0\}$; in fact, in this case $Q \in \mathbb{R}^{n \times n}$ leads to $U=0$. With reference to the discussion in Section 2, note that with $S=\left[\begin{array}{c}S_{c} \\ Q\end{array}\right]$, where the rows of $S_{c}$ are linearly independent from those of $Q$, we have that for all $i \in\{1,2\}$

$$
S\left(A_{i}+G_{i} C\right) S^{-1}=\left[\begin{array}{cc}
\Delta_{i}^{11}(K, U) & \Delta_{i}^{12}(K, U) \\
0 & \Delta_{i}^{22}(K, U)
\end{array}\right] .
$$

Equation (17) expresses the fact that, as repeatedly mentioned, $\mathcal{S}$ is an $\left(A_{1}+G_{1} C, A_{2}+G_{2} C\right)$ invariant subspace. The dependence of matrices $\Delta_{i}^{11}, \Delta_{i}^{12}$ and $\Delta_{i}^{22}$ upon $U$ and $K$ expresses the fact that $U$ and $K$ are the two degrees of freedom that can be used to assign the inner dynamics of $\mathcal{S}$ by modifying $\Delta_{i}^{11}(K, U)$ and to assign the external dynamics of $\mathcal{S}$ by modifying $\Delta_{i}^{22}(K, U)$. Importantly, these two procedures can be carried out independently; in fact, as the following lemma explains, the choice of $K$ affects $\Delta_{i}^{22}(K, U)$ but not $\Delta_{i}^{11}(K, U), i \in\{1,2\}$. Vice-versa, the choice of $U$ affects $\Delta_{i}^{11}(K, U)$ but not $\Delta_{i}^{22}(K, U), i \in\{1,2\}$.

Lemma 3.2 For all $i \in\{1,2\}$, the matrix $\Delta_{i}^{22}(K, U)$ in (17) does not depend on $U$, and the matrix $\Delta_{i}^{11}(K, U)$ does not depend on $K$.

Proof: First, we show that $\Delta_{i}^{(22)}(K, U)$ does not depend on $U$. To this end, let $U_{1}, U_{2}$ be two arbitrary matrices of suitable size. From (17) we find that

$$
\begin{aligned}
& {\left[\begin{array}{cc}
\Delta_{i}^{11}\left(K, U_{1}\right)-\Delta_{i}^{11}\left(K, U_{2}\right) & \Delta_{i}^{12}\left(K, U_{1}\right)-\Delta_{i}^{12}\left(K, U_{2}\right) \\
0 & \Delta_{i}^{22}\left(K, U_{1}\right)-\Delta_{i}^{22}\left(K, U_{2}\right)
\end{array}\right]} \\
& \quad=S\left(A_{i}+G_{\Lambda} C+\Omega U_{1} C\right) S^{-1}-S\left(A_{i}+G_{\Lambda} C+\Omega U_{2} C\right) S^{-1}=\left[\begin{array}{c}
S_{c} \\
Q
\end{array}\right] \Omega\left(U_{1}-U_{2}\right) C S^{-1},
\end{aligned}
$$

so that

$$
Q \Omega\left(U_{1}-U_{2}\right) C S^{-1}=\left[\begin{array}{ll}
0 & \Delta_{i}^{22}\left(K, U_{1}\right)-\Delta_{i}^{22}\left(K, U_{2}\right)
\end{array}\right]
$$

which is equal to zero since $Q \Omega$ is the zero matrix by definition of $\Omega$. Hence, $\Delta_{i}^{22}\left(K, U_{1}\right)=\Delta_{i}^{22}\left(K, U_{2}\right)$, which implies that the term $\Delta_{i}^{22}(K, U)$ in (17) does not depend on $U$. Now we show that $\Delta_{i}^{11}(K, U)$ does not depend on $K$. First, from (17), it is found that

$$
S_{c}\left(A_{i}-Q^{\top}\left(Q Q^{\top}\right)^{-1} \Lambda C+\Omega U C\right)=\Delta_{i}^{11}(K, U) S_{c}+\Delta_{i}^{12}(K, U) Q
$$

where $U$ is an arbitrary matrix of suitable size. Let $\Gamma_{1}$ and $\Lambda_{1}$ be the solution of (14) with $K=K_{1}$, and let $\Gamma_{2}$ and $\Lambda_{2}$ be the solution of (14) with $K=K_{2}$. Since $Q A_{H}=\Gamma_{j} Q_{D}+\Lambda_{j} C_{D}$, for $j \in\{1,2\}$, we get $\left(\Lambda_{1}-\Lambda_{2}\right) C_{D}=-\left(\Gamma_{1}-\Gamma_{2}\right) Q_{D}$. By partitioning $\left(\Gamma_{1}-\Gamma_{2}\right)$ as $\left[\Xi_{1} \Xi_{2}\right]$, we get $\left(\Lambda_{1}-\Lambda_{2}\right) C=-\Xi_{i} Q$. Writing (18) with respect to $\Lambda_{1}$ and $\Lambda_{2}$ and by computing the difference yields

$$
S_{c}\left(-Q^{\top}\left(Q Q^{\top}\right)^{-1}\left(\Lambda_{1}-\Lambda_{2}\right) C\right)=\left(\Delta_{i}^{11}\left(K_{1}, U\right)-\Delta_{i}^{11}\left(K_{2}, U\right)\right) S_{c}+\left(\Delta_{i}^{12}\left(K_{1}, U\right)-\Delta_{i}^{12}\left(K_{2}, U\right)\right) Q,
$$


so that

$$
S_{c} Q^{\top}\left(Q Q^{\top}\right)^{-1} \Xi_{i} Q=\left(\Delta_{i}^{11}\left(K_{1}, U\right)-\Delta_{i}^{11}\left(K_{2}, U\right)\right) S_{c}+\left(\Delta_{i}^{12}\left(K_{1}, U\right)-\Delta_{i}^{12}\left(K_{2}, U\right)\right) Q .
$$

Since $Q$ and $S_{c}$ have linearly independent rows, we find

$$
S_{c} Q^{\top}\left(Q Q^{\top}\right)^{-1} \Xi_{i} Q=\left(\Delta_{i}^{12}\left(K_{1}, U\right)-\Delta_{i}^{12}\left(K_{2}, U\right)\right) Q
$$

and

$$
\left(\Delta_{i}^{11}\left(K_{1}, U\right)-\Delta_{i}^{11}\left(K_{2}, U\right)\right) S_{c}=0
$$

the second yielding $\Delta_{i}^{11}\left(K_{1}, U\right)=\Delta_{i}^{11}\left(K_{2}, U\right)$ since $S_{c}$ has linearly independent rows. Therefore, the term $\Delta_{i}^{11}(K, U)$ in (17) does not depend on $K$.

Now we want to find a method to design the output-injection matrix $G=\left[\begin{array}{ll}G_{1} & G_{2}\end{array}\right]$ such that $\mathcal{S}$ is an externally stable $\left(A_{1}+G_{1} C, A_{2}+G_{2} C\right)$-invariant subspace; i.e., such that there exists an asymptotically stable pair $\left(\Gamma_{1}, \Gamma_{2}\right)$ for which $Q\left(A_{H}+G C_{D}\right)=\Gamma Q_{D}$.

For a given a conditioned invariant $\mathcal{S}$, write $(14)$ as

$$
\left[\begin{array}{lll}
\Gamma_{1} & \Gamma_{2} & \Lambda
\end{array}\right]=\left[\begin{array}{lll}
V_{1} & V_{2} & \bar{V}
\end{array}\right]+K\left[\begin{array}{lll}
H_{1} & H_{2} & \bar{H}
\end{array}\right]
$$

where $\left[\begin{array}{lll}V_{1} & V_{2} & \bar{V}\end{array}\right]=Q A_{H}\left[\begin{array}{l}Q_{D} \\ C_{D}\end{array}\right]^{\dagger}$ and $\left[\begin{array}{lll}H_{1} & H_{2} & \bar{H}\end{array}\right]=H$ are partitioned comformably with $\left[\begin{array}{lll}\Gamma_{1} & \Gamma_{2} & \Lambda\end{array}\right]$, i.e., $\Gamma_{i}=V_{i}+K H_{i}$ for $i=1,2$ and $\Lambda=\bar{V}+K \bar{H}$. If $\mathcal{S}_{D}+\operatorname{ker} C_{D}=\mathbb{R}^{2 n}$, there is only one solution to (13), so that there are no degrees of freedom in the choice of the pair $\left(\Gamma_{1}, \Gamma_{2}\right)$. In this case, if $\left(\Gamma_{1}, \Gamma_{2}\right)$ is asymptotically stable, then with the corresponding $\Lambda=\bar{V}$, the matrix $G_{\Lambda} \stackrel{\text { def }}{=}-Q^{\top}\left(Q Q^{\top}\right)^{-1} \Lambda=\left[\begin{array}{ll}G_{\Lambda, 1} & G_{\Lambda, 2}\end{array}\right]$ is such that $\mathcal{S}$ is an externally stable $\left(A_{1}+G_{\Lambda, 1} C, A_{2}+G_{\Lambda, 2} C\right)$ invariant subspace. On the other hand, if the pair $\left(\Gamma_{1}, \Gamma_{2}\right)$ is not asymptotically stable, the subspace $\mathcal{S}$ is not externally stabilisable.

Now, when $\mathcal{S}_{D}+\operatorname{ker} C_{D} \subset \mathbb{R}^{2 n}$, the problem we need to solve is to find a matrix $K$ such that the resulting pair $\left(\Gamma_{1}, \Gamma_{2}\right)=\left(V_{1}+K H_{1}, V_{2}+K H_{2}\right)$ is asymptotically stable; the corresponding $\Lambda=\bar{V}+K \bar{H}$, for which $(\Gamma, \Lambda)$ is a solution of (11), is such that $G_{\Lambda} \stackrel{\text { def }}{=}-Q^{\top}\left(Q Q^{\top}\right)^{-1} \Lambda$, yielding $Q\left(A_{H}+G_{\Lambda} C_{D}\right)=\Gamma Q_{D}$, so that $\mathcal{S}$ is an externally stable $\left(A_{1}+G_{\Lambda, 1} C, A_{2}+G_{\Lambda, 2} C\right)$-invariant subspace.

Towards characterising a subset of such matrices $K$, we can virtually exploit any stability criterion for 2-D Fornasini-Marchesini models. As mentioned, necessary and sufficient conditions have recently appeared in the literature that characterise stability in finite terms, [12, 34]. For the sake of simplicity, however, we consider the sufficient condition recalled in Lemma 2.2, whose structure appears to be much less involved. Let us rewrite this condition for asymptotic stability in Lemma 2.2 for the pair $\left(\Gamma_{1}, \Gamma_{2}\right)$ as

$$
\left[\begin{array}{cc}
\Phi & 0 \\
0 & \Psi-\Phi
\end{array}\right]-\left[\begin{array}{c}
\Gamma_{1}^{\top} \\
\Gamma_{2}^{\top}
\end{array}\right] \Psi\left[\begin{array}{cc}
\Gamma_{1} & \Gamma_{2}
\end{array}\right]>0
$$


for some $\Phi \stackrel{\text { def }}{=} P_{1}>0$ and $\Psi \stackrel{\text { def }}{=} P_{1}+P_{2}>0$. Standard manipulation and $\Gamma_{i}=V_{i}+K H_{i}$, for $i=1,2$, yield the equivalent condition

$$
\left[\begin{array}{ccc}
\Phi & 0 & \left(\Psi V_{1}+\Theta H_{1}\right)^{\top} \\
0 & \Psi-\Phi & \left(\Psi V_{2}+\Theta H_{2}\right)^{\top} \\
\Psi V_{1}+\Theta H_{1} & \Psi V_{2}+\Theta H_{2} & \Psi
\end{array}\right]>0
$$

for some $\Phi>0, \Psi>0$, and $\Theta$ of suitable dimensions, where $\Theta=\Psi K$. We have just proved the following result

Theorem 3.1 Let $\mathcal{S}$ be a conditioned invariant subspace for (1). Then, $\mathcal{S}$ is an externally stabilisable conditioned invariant subspace if there exist $\Phi=\Phi^{\top}>0, \Psi=\Psi^{\top}>0$ and $\Theta$ of suitable dimensions such that (20) holds. Moreover, given a triple $(\Theta, \Phi, \Psi)$ in the convex set defined by (20), a matrix $K$ for which the pair $\left(\Gamma_{1}, \Gamma_{2}\right)$ is asymptotically stable is given by $K=\Psi^{-1} \Theta$.

\section{Detectability Subspaces and Local State Observers}

Now we turn our attention to input-containing subspaces, which are particular types of conditionedinvariant subspaces. These are useful for various filtering/estimation problems, including the construction of local state observers without access to the system inputs.

Definition 4.1 We define an input-containing subspace $\mathcal{S}$ for (1) as a subspace of $\mathbb{R}^{n}$ such that

$$
\left[\begin{array}{ll}
A_{H} & B_{H}
\end{array}\right]\left(\left(\mathcal{S}_{D} \oplus \mathbb{R}^{2 m}\right) \cap \operatorname{ker}\left[\begin{array}{ll}
C_{D} & D_{D}
\end{array}\right]\right) \subseteq \mathcal{S}
$$

As for the 1-D case, it is easy to see that the intersection of two input-containing subspaces is input-containing. It follows that the set of input-containing subspaces for (1) is closed under subspace intersection. The same is not true for subspace addition. This is due to the fact that the Grassman manifold of $\mathbb{R}^{n}$ is a non-distributive lattice with respect to the operations of sum and intersection (and with respect to the partial ordering given by the standard subspace inclusion $\subseteq$ ), [3]. As a result of these considerations, it turns out that the set of input-containing subspaces for (1) is a modular lower semilattice with respect to subspace intersection. Thus, the intersection of all input-containing subspaces of $\Sigma$ is the smallest input-containing subspace of $\Sigma$, and is usually denoted by $\mathcal{S}^{\star}$. A simple algorithm for the computation of $\mathcal{S}^{\star}$ is given below. This algorithm extends Proposition 3.4 in [9] to non-strictly proper systems.

Algorithm 4.1 The sequence of subspaces $\left(\mathcal{S}^{i}\right)_{i \in \mathbb{N}}$ described by the recurrence

$$
\begin{aligned}
& \mathcal{S}^{0}=0_{n} \\
& \mathcal{S}^{i}=\left[\begin{array}{ll}
A_{H} & B_{H}
\end{array}\right]\left(\left(\mathcal{S}_{D}^{i-1} \oplus \mathbb{R}^{3 m}\right) \cap \operatorname{ker}\left[\begin{array}{ll}
C_{D} & D_{D}
\end{array}\right]\right),
\end{aligned}
$$

for $i>0$, is monotonically non-increasing. An integer $k \leq n-1$ exists such that $\mathcal{S}^{k+1}=\mathcal{S}^{k}$. For such $k$, the identity $\mathcal{S}^{\star}=\mathcal{S}^{k}$ holds. 
For input-containing subspaces, a generalised version of Lemma 3.1 holds.

Lemma 4.1 Given the $s$-dimensional subspace $\mathcal{S}$ of $\mathbb{R}^{n}$, let $Q \in \mathbb{R}^{(n-s) \times n}$ be a full row-rank matrix such that $\operatorname{ker} Q=\mathcal{S}$. The following statements are equivalent:

(i) the subspace $\mathcal{S}$ is input-containing for (1);

(ii) two matrices $\Gamma \in \mathbb{R}^{(n-s) \times 2(n-s)}$ and $\Lambda \in \mathbb{R}^{(n-s) \times 2 p}$ exist such that

$$
Q\left[\begin{array}{ll}
A_{H} & B_{H}
\end{array}\right]=\Gamma\left[\begin{array}{ll}
Q_{D} & 0_{2(n-s) \times 2 m}
\end{array}\right]+\Lambda\left[\begin{array}{ll}
C_{D} & D_{D}
\end{array}\right]
$$

(iii) a matrix $G \in \mathbb{R}^{n \times 2 p}$ exists such that

$$
\left[A_{H}+G C_{D} \quad B_{H}+G D_{D}\right]\left(\mathcal{S}_{D} \oplus \mathbb{R}^{2 m}\right) \subseteq \mathcal{S}
$$

Proof: The result follows in the same way as the result in Lemma 3.1.

Following the procedure outlined for 2-D conditioned invariant subspaces, to find the set of ouputinjection matrices associated with the input-containing subspace $\mathcal{S}$, we first solve (21) with respect to $\Gamma$ and $\Lambda$, obtaining

$$
\left[\begin{array}{ll}
\Gamma & \Lambda
\end{array}\right]=Q\left[\begin{array}{ll}
A_{H} & B_{H}
\end{array}\right]\left[\begin{array}{cc}
Q_{D} & 0 \\
C_{D} & D_{D}
\end{array}\right]^{\dagger}+K H
$$

where $H$ is full row-rank, ker $H=\operatorname{im}\left[\begin{array}{cc}Q_{D} & 0 \\ C_{D} & D_{D}\end{array}\right]$, and $K$ is an arbitrary matrix of suitable size. Using (22), we compute the solutions of $\Lambda=-Q G$ as $G=G_{\Lambda}+\Omega U$. As for conditioned invariant subspaces, $K$ represents the degree of freedom that can be used to assign the external dynamics of the inputcontaining subspace $\mathcal{S}$, e.g. by means of an LMI condition similar to that given in Theorem 3.1. As such, we say that $\mathcal{S}$ is a detectability subspace if an output-injection matrix $G$ exists (or, equivalently, if $K$ exists) such that (22) holds and $\mathcal{S}$ is an externally stable $\left(A_{1}+G_{1} C, A_{2}+G_{2} C\right)$-invariant subspace. It can be straightforwardly established that an exact equivalent of Theorem 3.1 holds for detectability subspaces by simply writing (19) with

$$
\left[\begin{array}{lll}
V_{1} & V_{2} & \bar{V}
\end{array}\right]=Q\left[\begin{array}{ll}
A_{H} & B_{H}
\end{array}\right]\left[\begin{array}{cc}
Q_{D} & 0 \\
C_{D} & D_{D}
\end{array}\right]^{\dagger} \quad \text { and } \quad \operatorname{ker}\left[\begin{array}{ccc}
H_{1} & H_{2} & \bar{H}
\end{array}\right]=\operatorname{im}\left[\begin{array}{cc}
Q_{D} & 0 \\
C_{D} & D_{D}
\end{array}\right]
$$

Detectability input-containing subspaces can be linked to the existence of certain observers [28]. Consider a system $\Sigma$ governed by a Fornasini-Marchesini model (1). Given a subspace $\mathcal{S}$ of $\mathbb{R}^{n}$, the 2-D system $\Sigma_{\mathrm{O}}$ ruled by $(2)$ is said to be an $\mathcal{S}$-quotient observer if for any boundary condition of $\Sigma$ and $\Sigma_{O}$, the local state of $\Sigma_{O}$ asymptotically reconstructs the local state $x_{i, j}$ of $\Sigma$ modulo the components of this vector on $\mathcal{S}$. In other words, on the basis of the observations $y$, the vector $\omega_{i, j}$ asymptotically converges to $x_{i, j} / \mathcal{S}$, as the indexes $i$ and $j$ evolve away from the boundary, regardless of the boundary conditions of $\Sigma$ and $\Sigma_{\mathrm{O}}$. 
Obviously, given an arbitrary subspace $\mathcal{S}$ of $\mathbb{R}^{n}$, an $\mathcal{S}$-quotient observer does not necessarily exists. But if this subspace is a detectability subspace, the existence of such an observer is guaranteed. Let $Q$ be a full row-rank matrix such that $\operatorname{ker} Q=\mathcal{S}$. Define the new variable $e_{i, j}=Q x_{i, j}-\omega_{i, j}$, along with the vectors $\hat{x}(i, j)=\left[\begin{array}{lll}x_{i, j}^{\top} & x_{i+1, j}^{\top} & x_{i, j+1}^{\top}\end{array}\right]^{\top}, \hat{u}(i, j)=\left[\begin{array}{lll}u_{i, j}^{\top} & u_{i+1, j}^{\top} & u_{i, j+1}^{\top}\end{array}\right]^{\top}$ and $\hat{\omega}(i, j)=\left[\begin{array}{lll}\omega_{i, j}^{\top} & \omega_{i+1, j}^{\top} & \omega_{i, j+1}^{\top}\end{array}\right]^{\top},(i, j) \in \mathbb{S}_{+}$. Let $\Gamma$ and $\Lambda$ be such that (21) holds. Let system (2) be defined by $K_{H}=\Gamma$ and $L_{H}=\Lambda$. It is found that

$$
\begin{aligned}
e_{i+1, j+1} & =Q x_{i+1, j+1}-\omega_{i+1, j+1} \\
& =Q A_{H} \hat{x}(i, j)+Q B_{H} \hat{u}(i, j)-\Gamma \hat{\omega}(i, j)-Q G C_{D} \hat{x}(i, j)-Q G D_{D} \hat{u}(i, j) \\
& =Q\left[A_{H}+G C_{D} B_{H}+G D_{D}\right]\left[\begin{array}{l}
\hat{x}(i, j) \\
\hat{u}(i, j)
\end{array}\right]-\Gamma \hat{\omega}(i, j)=\Gamma_{1} e_{i+1, j}+\Gamma_{2} e_{i, j+1},
\end{aligned}
$$

where (22) has been used. Moreover, since $\mathcal{S}$ is a detectability subspace, the pair $\left(\Gamma_{1}, \Gamma_{2}\right)$ is asymptotically stable. Therefore, the estimation error converges to zero as the index $(i, j)$ evolves away from $\mathbb{S}_{0}$, so that $\omega_{i, j}$ asymptotically converges to $Q x_{i, j}$. Since ker $Q=\mathcal{S}$, this means that $\Sigma_{\mathrm{O}}$ recovers the external components of $x_{i, j}$ with respect to $\mathcal{S}$. Notice that if $\mathcal{S}$ is an input-containing subspace but not a detectability subspace, the estimation error does not converge to zero, unless the error is zero over the entire boundary region.

Note that the characterisation of external stabilisability for conditioned invariant and inputcontaining subspaces is essential in employing these ideas in the construction of local state observers. Indeed, the fact that the subspace $\mathcal{S}$ is input-containing alone can only guarantee that $\Sigma_{\mathrm{O}}$ gives rise to an estimation error that only depends on the boundary conditions. Therefore, $\Sigma_{\mathrm{O}}$ can only guarantee that when $\omega_{i, j}=Q x_{i, j}$ for $(i, j) \in \mathbb{S}_{0}$, then the estimation error is identically zero, which means that $\omega_{i, j}=Q x_{i, j}$ for all $\mathbb{S}_{+}$.

Example 4.1 Consider (1) with

$$
\begin{aligned}
& A_{1}=\left[\begin{array}{cccc}
-0.35 & 0 & 0.01 & 0 \\
0 & 0.1 & 0 & 0 \\
0.01 & 0 & -0.4 & 0.4 \\
0 & 0 & 0.02 & 0.15
\end{array}\right], \quad A_{2}=\left[\begin{array}{cccc}
0 & 0 & -0.05 & 0 \\
0.5 & 0.01 & 0 & 0 \\
-0.35 & 0 & 0.35 & 0.05 \\
0 & 0 & -0.45 & 0.15
\end{array}\right], \quad B_{1}=\left[\begin{array}{cc}
0 & 0 \\
1 & 6 \\
0 & -5 \\
0 & 2
\end{array}\right], \quad B_{2}=\left[\begin{array}{cc}
0 & 2 \\
-7 & 0 \\
0 & 0 \\
0 & 0
\end{array}\right], \\
& C=\left[\begin{array}{cccc}
0.4 & 0 & 0 & 0 \\
0 & 0 & 0 & -2
\end{array}\right], \quad D=\left[\begin{array}{cc}
0 & 2 \\
0 & -7
\end{array}\right] .
\end{aligned}
$$

Using Algorithm 4.1, which in this case converges in one step, it is found that $\mathcal{S}^{\star}=\operatorname{im}\left[\begin{array}{llll}0 & 1 & 0 & 0\end{array}\right]^{\top}$, so that the kernel of

$$
Q=\left[\begin{array}{llll}
1 & 0 & 0 & 0 \\
0 & 0 & 1 & 0 \\
0 & 0 & 0 & 1
\end{array}\right]
$$


is exactly $\mathcal{S}^{\star}$. By defining $W \stackrel{\text { def }}{=}\left[\begin{array}{cc}Q_{D} & 0 \\ C_{D} & D_{D}\end{array}\right]$, a matrix $H$ whose kernel is the image of $W$ can be easily computed:

$$
H=\left[\begin{array}{cccccccccc}
1 & 0 & -\frac{10}{7} & 0 & 0 & 0 & -\frac{5}{2} & -\frac{5}{7} & 0 & 0 \\
0 & 0 & 0 & 1 & 0 & -\frac{10}{7} & 0 & 0 & \frac{5}{2} & -\frac{5}{7}
\end{array}\right] .
$$

Using (23), it is easy to verify - for example exploiting the MATLAB ${ }^{\circledR}$ routines setlmis.m, Imiterm.m, getlmis.m and feasp.m - that the LMI condition (20) is satisfied with

$$
\Phi=\left[\begin{array}{ccc}
3.6255 & -0.3427 & 0.3978 \\
-0.3427 & 0.4365 & 0.2150 \\
0.3978 & 0.2150 & 1.0328
\end{array}\right], \quad \Psi=\left[\begin{array}{ccc}
5.4473 & -0.1769 & 0.6142 \\
-0.1769 & 1.2425 & 0.9384 \\
0.6142 & 0.9384 & 2.4452
\end{array}\right],
$$

which are symmetric and positive definite, and

$$
\Theta=\left[\begin{array}{cc}
-0.1101 & -0.9151 \\
0.9581 & 0.1717 \\
0.4129 & 0.4502
\end{array}\right] \text {, which yields } K=\Psi^{-1} \Theta=\left[\begin{array}{cc}
0.0314 & -0.2017 \\
0.9209 & -0.0956 \\
-0.1925 & 0.2715
\end{array}\right] .
$$

As such,

$$
\left[\begin{array}{l|l}
\Gamma_{1} & \Gamma_{2}
\end{array}\right]=\left[\begin{array}{ccc|ccc}
-0.2829 & 0.0100 & -0.0959 & -0.3058 & -0.0500 & -0.1346 \\
1.2485 & -0.4000 & 0.0593 & -0.4026 & 0.3500 & 0.1252 \\
-0.2747 & 0.0200 & -0.0290 & 0.2934 & -0.4500 & -0.2691
\end{array}\right]
$$

and

$$
\left[\begin{array}{l|l}
\Lambda_{1} & \Lambda_{2}
\end{array}\right]=\left[\begin{array}{cc|cc}
-0.1678 & -0.0479 & 0.7645 & -0.0673 \\
-3.0962 & -0.1703 & 0.1315 & 0.0376 \\
0.6868 & -0.0895 & -0.7334 & -0.2095
\end{array}\right]
$$

The observer (2) with $K_{1}=\Gamma_{1}, K_{2}=\Gamma_{2}, L_{1}=\Lambda_{1}$ and $L_{2}=\Lambda_{2}$, whose order is 3 , is able to reconstruct the local state of the system modulo $\mathcal{S}^{\star}$, i.e., the first, the third and the fourth components, but not the second. Indeed, by denoting by $x_{i, j}^{(k)}$ the $k$-the component of vector $x_{i, j}$, the estimation error is

$$
e_{i, j}=Q x_{i, j}-\omega_{i, j}=\left[\begin{array}{c}
x_{i, j}^{(1)}-\omega_{i, j}^{(1)} \\
x_{i, j}^{(3)}-\omega_{i, j}^{(2)} \\
x_{i, j}^{(4)}-\omega_{i, j}^{(3)}
\end{array}\right] .
$$

If for simplicity of representation we consider as boundary the set of indexes $(\{0\} \times[1,20]) \cup([1,20] \times$ $\{0\}$ ), and we assign random boundary conditions (using randn.m) on this region, using the randomly generated input functions of Figure 1, we obtain that the estimation error is the one given in Figure 2. Notice that indeed the estimation errors converge to zero as the index $(i, j)$ moves away from the boundary. 

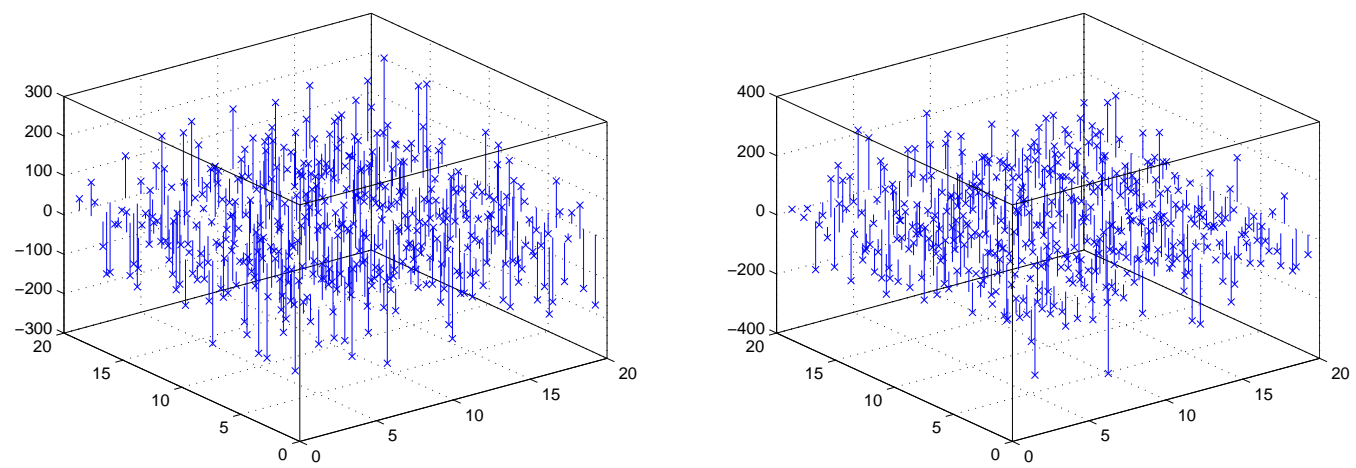

Figure 1: Inputs $u^{1}$ and $u^{2}$ in the bounded frame $[0,20] \times[0,20]$.
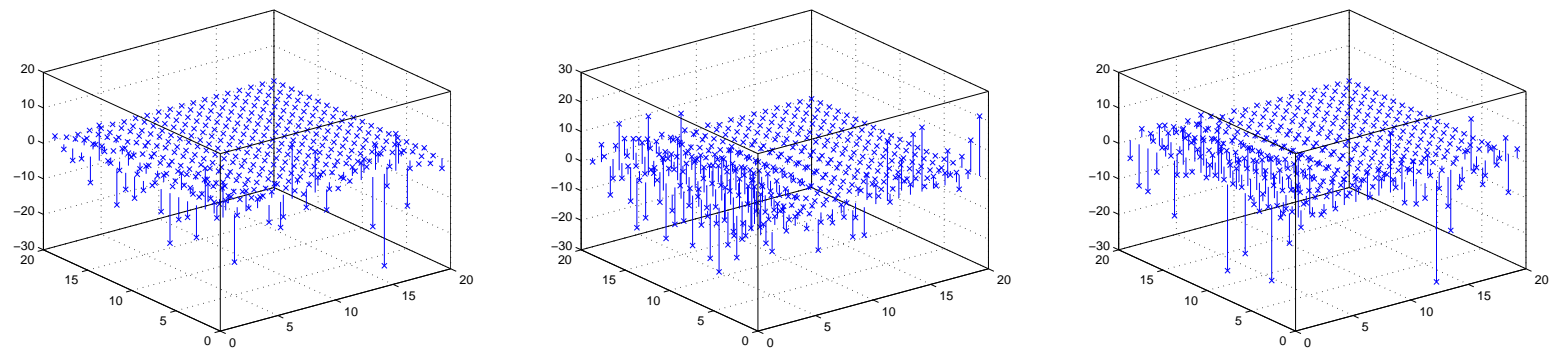

Figure 2: Estimation errors over the bounded frame $[0,20] \times[0,20]$.

\section{Unknown-input observers}

In this section, we use the geometric notions developed so far for the solution of the unknown-input observation problem, which plays an important role in signal reconstruction problems, fault-detection and identification, non-interaction control. Consider the Fornasini-Marchesini model

$$
\begin{aligned}
x_{i+1, j+1} & =A_{1} x_{i+1, j}+A_{2} x_{i, j+1}+B_{1} u_{i+1, j}+B_{2} u_{i, j+1} \\
y_{i, j} & =C x_{i, j}+D u_{i, j} \\
z_{i, j} & =R x_{i, j}+S u_{i, j}
\end{aligned}
$$

where, for all intgers $i, j$, vector $u_{i, j} \in \mathbb{R}^{m}$ represents an input which is not accessible for measurement. The variable $y_{i, j} \in \mathbb{R}^{p_{1}}$ represents an output that can be measured and the variable $z_{i, j} \in \mathbb{R}^{p_{2}}$ is an output that we want to estimate on the basis of the measurement $y$. All matrices appearing in (24) are of appropriate dimensions. Consider the block diagram depicted in Figure 3. Let the observer $\Sigma_{\mathrm{O}}$ be described by the equations

$$
\begin{aligned}
\omega_{i+1, j+1} & =K_{1} \omega_{i+1, j}+K_{2} \omega_{i, j+1}+L_{1} y_{i+1, j}+L_{2} y_{i, j+1}, \\
\zeta_{i, j} & =M \omega_{i, j}+N y_{i, j}
\end{aligned}
$$


and let $\widehat{\Sigma}$ denote the overall system from the input $u$ to the output $e:=z-\zeta$. With the choice of the structure of the observer $\Sigma_{\mathrm{O}}$, the overall system is governed by

$$
\begin{aligned}
& {\left[\begin{array}{l}
x_{i+1, j+1} \\
\omega_{i+1, j+1}
\end{array}\right]=\left[\begin{array}{cc}
A_{1} & 0 \\
L_{1} C & K_{1}
\end{array}\right]\left[\begin{array}{l}
x_{i+1, j} \\
\omega_{i+1, j}
\end{array}\right]+\left[\begin{array}{cc}
A_{2} & 0 \\
L_{2} C & K_{2}
\end{array}\right]\left[\begin{array}{l}
x_{i, j+1} \\
\omega_{i, j+1}
\end{array}\right]} \\
& +\left[\begin{array}{c}
B_{1} \\
L_{1} D
\end{array}\right] u_{i+1, j}+\left[\begin{array}{c}
B_{2} \\
L_{2} D
\end{array}\right] u_{i, j+1}, \\
& e_{i, j}=[R-N C-M]\left[\begin{array}{l}
x_{i, j} \\
\omega_{i, j}
\end{array}\right]+(S-N D) u_{i, j} \text {. }
\end{aligned}
$$

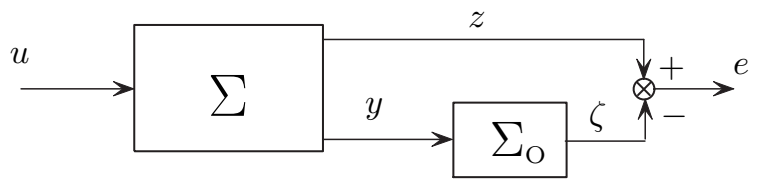

Figure 3: Block diagram of the unknown input observation scheme.

Roughly speaking, the unknown-input observation problem consists of finding $\Sigma_{\mathrm{O}}$ ruled by (25) and connected as in Figure 3, such that $\Sigma_{\mathrm{O}}$ recovers the local state $x_{i, j}$ with greater accuracy as the spatial index $(i, j)$ evolves away from $\mathbb{S}_{0}$, i.e., such that for any boundary conditions of $\Sigma$ and $\Sigma_{\mathrm{O}}$ the estimation error $e_{i, j}$ converges to zero as $(i, j)$ evolves away from $\mathbb{S}_{0}$. This problem is equivalent to finding an observer $\Sigma_{\mathrm{O}}$ such that the input $u$ has no influence on the output $e$. The case in which the observer is dead-beat, i.e., in which the estimation error goes to zero within a finite number of steps for any boundary conditions of $\Sigma$ and $\Sigma_{\mathrm{O}}$, was completely solved in [5] using polynomial techniques. In the following theorem, a solution is provided for the unknown-input observation problem when only asymptotic convergence to zero of the estimation error is required. The solution is constructive, in the sense that a sufficient solvability condition is presented that guarantees the existence of an unknown-input observer that provides an asymptotic estimate of $z$. The observer model matrices are explicitly derived.

Theorem 5.1 Let $\mathcal{S}^{\star}$ be the smallest input-containing subspace of the Fornasini-Marchesini model

(1). The unknown-input observation problem admits solutions if

(i) $\operatorname{ker}[R \quad S] \supseteq\left(\mathcal{S}^{\star} \oplus \mathbb{R}^{m}\right) \cap \operatorname{ker}\left[\begin{array}{ll}C & D\end{array}\right]$;

(ii) $\mathcal{S}^{\star}$ is a detectability subspace.

Proof: Let $Q$ be a full row-rank matrix such that $\operatorname{ker} Q=\mathcal{S}^{\star}$. Condition (i) implies that a pair $(\Phi, \Psi)$ exists such that

$$
\left[\begin{array}{ll}
R & S
\end{array}\right]=\Phi\left[\begin{array}{ll}
Q & 0
\end{array}\right]+\Psi\left[\begin{array}{ll}
C & D
\end{array}\right]
$$


The solutions $\Phi$ and $\Psi$ of (26) are parameterised as

$$
\left[\begin{array}{ll}
\Phi & \Psi
\end{array}\right]=\left[\begin{array}{ll}
R & S
\end{array}\right]\left[\begin{array}{ll}
Q & 0 \\
C & D
\end{array}\right]^{\dagger}+K H
$$

where the rows of $H$ span the null-space of $\left[\begin{array}{cc}Q^{\top} & C^{\top} \\ 0 & D^{\top}\end{array}\right]$ and $K$ is an arbitrary matrix of suitable size. Furthermore, the matrices $\Phi$ and $\Psi$ satisfying (26) are unique if and only if the map $\left[\begin{array}{ll}Q & 0 \\ C & D\end{array}\right]$ is surjective. When $\left[\begin{array}{ll}C & D\end{array}\right]$ is full row-rank, this condition is equivalent to $C \mathcal{S}^{\star}+\operatorname{im} D=\mathbb{R}^{p_{1}}$ or alternatively $\mathcal{S}^{\star}+C^{-1} \mathrm{im} D=\mathbb{R}^{n} .^{2}$ Notice also that equation (26) can be equivalently written as

$$
\left[\begin{array}{ll}
R_{D} & S_{D}
\end{array}\right]=\Phi_{D}\left[\begin{array}{ll}
Q_{D} & 0
\end{array}\right]+\Psi_{D}\left[\begin{array}{ll}
C_{D} & D_{D}
\end{array}\right]
$$

Now, since obviously the kernel of $\left[\begin{array}{cc}Q^{\top} & C^{\top} \\ 0 & D^{\top}\end{array}\right]$ is zero if and only if such is the kernel of $\left[\begin{array}{cc}Q_{D}^{\top} & C_{D}^{\top} \\ 0 & S_{D}^{\top}\end{array}\right]$, it turns out that in the case where $C \mathcal{S}^{\star}+\operatorname{im} D=\mathbb{R}^{p_{1}}$, equation

$$
Q\left[\begin{array}{ll}
A_{H} & B_{H}
\end{array}\right]=\Gamma\left[\begin{array}{ll}
Q_{D} & 0
\end{array}\right]+\Lambda\left[\begin{array}{ll}
C_{D} & D_{D}
\end{array}\right]
$$

admits a unique solution, so that the four matrices $\Gamma, \Lambda, \Phi$ and $\Psi$ can be uniquely determined. Since $\mathcal{S}^{\star}$ is a detectability subspace, there exists an output-injection matrix $G$ such that $(22)$ holds with an asymptotically stable pair $\left(\Gamma_{1}, \Gamma_{2}\right)$. We show that the dynamical system $\Sigma_{\mathrm{O}}$ ruled by $(25)$ with $K_{k}=\Gamma_{k}, L_{k}=-Q G_{k},(k \in\{1,2\}), M=\Phi$, and $N=\Psi$ solves the unknown-input observation problem. First, note that in view of (26)

$$
\begin{aligned}
e_{i, j} & =[R-N C-M]\left[\begin{array}{l}
x_{i, j} \\
\omega_{i, j}
\end{array}\right]+(S-N D) u_{i, j} \\
& =(R-\Psi C) x_{i, j}-\Phi \omega_{i, j}+(S-\Psi D) u_{i, j} \\
& =\left(\left[\begin{array}{ll}
R & S
\end{array}\right]-\Psi\left[\begin{array}{ll}
C & D
\end{array}\right]\right)\left[\begin{array}{c}
x_{i, j} \\
u_{i, j}
\end{array}\right]-\Phi \omega_{i, j} \\
& =\Phi\left[\begin{array}{ll}
Q & 0
\end{array}\right]\left[\begin{array}{l}
x_{i, j} \\
u_{i, j}
\end{array}\right]-\Phi \omega_{i, j}=\Phi\left(Q x_{i, j}-\omega_{i, j}\right) .
\end{aligned}
$$

Define $\varepsilon_{i, j}:=Q x_{i, j}-\omega_{i, j}$ using the same notation of Section 4 . Given the signal $s: \mathbb{S}_{+} \mapsto \mathbb{R}^{h}$ for some $h$, let also $\hat{s}(i, j) \stackrel{\text { def }}{=}\left[\begin{array}{lll}s_{i, j}^{\top} & s_{i+1, j}^{\top} & s_{i, j+1}^{\top}\end{array}\right]^{\top}$. It follows that

$$
\begin{aligned}
\varepsilon_{i+1, j+1}= & Q x_{i+1, j+1}-\omega_{i+1, j+1} \\
= & Q\left[\begin{array}{ll}
A_{H} & B_{H}
\end{array}\right]\left[\begin{array}{l}
\hat{x}(i, j) \\
\hat{u}(i, j)
\end{array}\right]-\Gamma \hat{\omega}(i, j) \\
& +Q G_{1}\left(C x_{i+1, j}+D u_{i+1, j}\right)+Q G_{2}\left(C x_{i, j+1}+D u_{i, j+1}\right) \\
= & Q\left(\left[\begin{array}{ll}
A_{H} & B_{H}
\end{array}\right]+G\left[\begin{array}{ll}
C_{D} & D_{D}
\end{array}\right]\right)\left[\begin{array}{l}
\hat{x}(i, j) \\
\hat{u}(i, j)
\end{array}\right]-\Gamma \hat{\omega}(i, j) \\
= & \Gamma\left[\begin{array}{ll}
Q & 0
\end{array}\right]\left[\begin{array}{l}
\hat{x}(i, j) \\
\hat{u}(i, j)
\end{array}\right]-\Gamma \hat{\omega}(i, j)=\Gamma_{1} \varepsilon_{i+1, j}+\Gamma_{2} \varepsilon_{i, j+1} .
\end{aligned}
$$

\footnotetext{
${ }^{2}$ Recall that $C^{-1}$ im $D=\left\{x \in \mathbb{R}^{n} \mid C x \in \operatorname{im} D\right\}$.
} 
Hence, the signal $\varepsilon_{i, j}$ is independent of $u_{i, j}$, and since $e_{i, j}=\Phi \varepsilon_{i, j}$, such is also the estimation error $e_{i, j}$. It follows that if $\zeta_{i, j}=z_{i, j}$ for all $(i, j) \in \mathbb{S}_{0}$, then $\zeta_{i, j}=z_{i, j}$ for all $(i, j) \in \mathbb{S}_{+}$. Moreover, in view of the asymptotic stability of the pair $\left(\Gamma_{1}, \Gamma_{2}\right)$, it also follows that for all boundary conditions $\zeta_{i, j}$ and $x_{i, j},(i, j) \in \mathbb{S}_{0}$, the estimation error $e_{i, j}$ converges to zero as $(i, j)$ moves away from $\mathbb{S}_{0}$.

\section{Concluding remarks}

The paper develops notions of conditioned invariant and detectability subspaces for 2-D FornasiniMarchesini models. By contrast with earlier work, the development here leads to an LMI based procedure for the synthesis of observers which asymptotically estimate the local state of a standard Fornasini-Marchesini model, in the sense that the error tends to zero as the reconstructed local state evolves away from unknown boundary conditions. The geometric notions and results presented here complement those in [27], where notions of controlled-invariance and stabilisability are developed within the context of 2-D disturbance decoupling problems. It is expect that the results of this paper will lead to 2-D extensions of techniques for the detection and identification of faults, as developed in $[25]$ and [31].

\section{References}

[1] G. Basile and G. Marro. Controlled and conditioned invariant subspaces in linear system theory. Journal of Optimization Theory and Applications, vol. 3, no. 5, pp. 306-315, May 1969.

[2] G. Basile and G. Marro. On the observability of linear, time-invariant systems with unknown inputs. Journal of Optimization Theory and Applications, vol. 3, no. 6, pp. 410-415, June 1969.

[3] G. Basile and G. Marro. Controlled and Conditioned Invariants in Linear System Theory. Prentice Hall, Englewood Cliffs, New Jersey, 1992.

[4] M. Bisiacco. On the structure of 2D observers. IEEE Transactions on Automatic Control, vol. AC-31, no. 7, pp. 676-680, July 1986.

[5] M. Bisiacco and M.E. Valcher. Unknown input observers for 2D state-space models. International Journal of Control, vol. 77, no. 9, pp. 861-876(16), June 10, 2004.

[6] M. Bisiacco and M.E. Valcher. Observer-based fault detection and isolation for 2D state-space models. Multidimensional Systems and Signal Processing, vol.17, pp. 219-242, 2006.

[7] M. Bisiacco and M.E. Valcher. The general fault detection and isolation problem for 2D statespace models. Systems \& Control Letters, vol.55, pp. 894-899, 2006.

[8] P.A. Bliman. Lyapunov Equation for the Stability of 2-D Systems. Multidimensional Systems and Signal Processing, vol. 13, pp. 201-222, 2002. 
[9] G. Conte and A. Perdon. On the geometry of 2D systems. Proc. IEEE Int. Symp. on Circuit and Systems, Helsinki, Finland, 1988.

[10] E. Fornasini and G. Marchesini. State-space realization theory of two-dimensional filters. IEEE Transactions on Automatic Control, vol. AC-21, no. 4, pp. 484-492, August 1976.

[11] E. Fornasini and G. Marchesini. Two dimensional filters: New aspects of the realization theory. Proceedings of the 3rd Conference on Pattern Recognition, Coronado, CA, 1976.

[12] Y. Ebihara, and Y. Ito, and T. Hagiwara, Exact Stability Analysis of 2-D Systems Using LMIs. IEEE Transactions on Automatic Control, vol. AC-51, no. 9, pp. 1509-1513, September 2006.

[13] E. Fornasini and G. Marchesini. Doubly-Indexed Dynamical Systems: State-Space Models and Structural Properties. Mathematical System Theory, vol. 12, pp. 59-72, 1978.

[14] E. Fornasini and G. Marchesini, Stability analysis of 2-D systems. IEEE Transactions on Circuits and Systems, CAS-27(12):1210-1217, 1980.

[15] G.D. Hu and M. Liu. Simple Criteria for Stability of Two-Dimensional Linear Systems. IEEE Transactions on Signal Processing, vol. 53, no. 12, pp. 4720-4723, December 2005.

[16] T. Kaczorek. The singular general model of 2-D systems and its solution. IEEE Transactions on Automatic Control, vol. AC-33, pp. 1060-1061, 1988.

[17] T. Kaczorek. Linear Control Systems. J.Wiley, NY, 1992.

[18] T. Kaczorek. Perfect observers for singular 2-D Fornasini-Marchesini models. IEEE Transactions on Automatic Control, vol. AC-40, pp. 1671-1675, 2001.

[19] H. Kar and V. Sigh. Stability of 2-D systems described by the Fornasini-Marchesini first model. IEEE Transactions on Signal Processing, vol. 51, no. 6, pp. 1675-1676, June 2003.

[20] A. Karamanciog̃lu and F.L. Lewis. A geometric approach to 2-D implicit systems. Proceedings of the 29th Conference on Decision and Control, Honolulu, Hawaii, December 1990.

[21] A. Karamanciog̃lu and F.L. Lewis. Geometric theory for the singular Roesser model. IEEE Transactions on Automatic Control, vol. AC-37, no. 6, pp. 801-806, June 1992.

[22] S.-Y. Kung, B.C. Levy, M. Morf, and T. Kailath, New results in 2-D systems theory, Part II: 2-D state-space models - realization and the notions of controllability, observability and minimality, Proceedings of the IEEE, vol. 65, no. 6, pp. 945-961, June 1977.

[23] J. Kurek. The general state-space model for a two-dimensional linear digital system. IEEE Transactions on Automatic Control, vol. AC-30, no. 6, pp. 600-602, June 1985.

[24] F.L. Lewis. A review of 2-D Implicit Systems. Automatica, vol. 28, no. 2, pp. 345-354, 1992. 
[25] M. Massoumnia. A geometric approach to the synthesis of failure detection filters. IEEE Transactions on Automatic Control, AC-31(9):839-846, September 1986.

[26] A.S. Morse. Structure and design of linear model following systems. IEEE Transactions on Automatic Control, vol. AC-18, no. 4, pp. 346-354, August 1973.

[27] L. Ntogramatzidis, M. Cantoni, and R. Yang, A geometric theory for 2-D systems including notions of stabilisability. Multidimensional Systems and Signal Processing, vol.19, pp. 449-475, 2008 .

[28] L. Ntogramatzidis and M. Cantoni, Conditioned invariance and unknown-input observation for two-dimensional Fornasini-Marchesini models. In Proceedings of European Control Conference, Kos, 2007.

[29] R.P. Roesser. A discrete state-space model for linear image processing. IEEE Transactions on Automatic Control, vol. AC-20, no. 1, pp. 1-10, February 1975.

[30] H.L. Trentelman, A.A. Stoorvogel, and M. Hautus. Control theory for linear systems. Communications and Control Engineering. Springer, Great Britain, 2001.

[31] Verghese G.C. Massoumnia, M. and A.S. Willsky. Failure detection and identification. IEEE Transactions on Automatic Control, AC-34(3):316-321, March 1989.

[32] J. Willems, Almost invariant subspaces: an approach to high gain feedback design - Part II: Almost conditionally invariant subspaces, IEEE Transactions on Automatic Control, vol. AC-26, no. 1, pp. 235-252, 1981.

[33] W.M. Wonham and A.S. Morse. Decoupling and pole assignment in linear multivariable systems: a geometric approach. SIAM Journal of Control, vol. 8, no. 1, pp. 1-18, 1970.

[34] T. Zhou. Stability and Stability Margin for a Two-Dimensional System. IEEE Transactions on Signal Processing, vol. 54, no. 9, pp. 3483-3488, September 2006. 